\title{
Die neue Stimme des fremden Helden: Gaetano Fraschini als Zamoro in Giuseppe Verdis Alzira
}

\author{
Thomas Seedorf
}

Obwohl es in der europäischen Musikgeschichte eine weit zurückreichende Tradition des musikalischen Exotismus gab, ${ }^{1}$ haben italienische Opernkomponisten der ersten Hälfte des 19. Jahrhunderts weitgehend darauf verzichtet, Schauplätze und Völker außereuropäischer Weltgegenden mit Hilfe exotistischer Topoi wie dem Gebrauch außergewöhnlicher Instrumente, besonderer Skalen oder spezifischer Rhythmen in ihrer Fremdheit ästhetisch zu vermitteln. Ein Grund für diese Zurückhaltung mag von politischen Zwängen beherrschten Pragmatik des Opernbetriebs dieser Zeit sein. Es kam häufig vor, dass die Autoren einer Oper ihr Werk auf Anweisung der Zensur, die stets bemüht war, politisch brisante Themen auf der Bühne zu unterdrücken, komplett umarbeiten mussten, oder Stücke an unterschiedlichen Orten in ganz unterschiedlichen Bearbeitungen aufgeführt wurden. Obwohl er bereits der berühmteste Komponist Italiens war, musste Giuseppe Verdi noch 1859 akzeptieren, dass die Handlung seiner Oper Un ballo in maschera vom Hof des schwedischen Königs Gustav III. nach Boston verlegt wurde - vergleichbare Fälle finden sich in der Geschichte der italienischen Oper des 19. Jahrhunderts zuhauf. Der Verzicht auf exotistische Darstellungsmittel erleichterte die Verlegung von Schauplätzen und entsprach damit der allgemeinen Bühnenpraxis.

Verdis Tragedia lirica Alzira spielt in Peru zur Zeit der Eroberung durch die Spanier. Der Komponist verzichtet zwar darauf, das Moment des Fremdkulturellen in seiner Musik zu spezifizieren, bringt aber dennoch ein neues und in gewisser Hinsicht fremd wirkendes Element zur Darstellung des Heroischen zur Geltung: einen neuen Sängertypus, dessen stimmliche Eigenheit Teil der Partitur ist.

Alzira entstand in einer stürmischen Phase der Laufbahn Giuseppe Verdis. ${ }^{2}$ Nach dem Erstling Oberto, Conte di San Bonifacio, der bei der Uraufführung 1839 am Mailänder Teatro alla Scala freundlich aufgenommenen wurde, und der komischen Oper Un giorno di regno, die im Folgejahr am selben Haus mit Pauken und Trompeten durchfiel, war Verdi 1842, wieder an der Scala, mit Nabucodonosor - besser bekannt unter der Kurzform Nabucco - der Durchbruch als Opernkomponist gelungen, der bald Anfragen anderer Opernhäuser Italiens nach sich zog. Ernani entstand 1844 als Auftragskomposition für das Teatro La Fenice in Venedig, noch im

1 Thomas Betzwieser und Michael Stegemann: Exotismus. In: Ludwig Finscher (Hg.): Die Musik in Geschichte und Gegenwart. Allgemeine Enzyklopädie der Musik, Sachteil, Bd. 3. ${ }^{2}$ Kassel [u.a.] 1995, Sp. 226-243.

2 Zu Verdis Laufbahn vgl. Julian Budden: Verdi. Leben und Werk. Stuttgart ${ }^{2} 2000$. 
selben Jahr folgte mit I due Foscari Verdis erste Oper für Rom. Mit Giovanna d'Arco kehrte Verdi 1845 noch einmal an die Scala zurück, ein knappes halbes Jahr später folgte die Uraufführung von Alzira am Teatro San Carlo in Neapel. Mit Attila, der 1846 am Teatro La Fenice erstmals gegeben wurde, schuf Verdi schließlich die fünfte Oper innerhalb von nur zwei Jahren - eine Tour de force, die den Komponisten in ganz Italien bekannt machte, ihm weitere Aufträge eintrug und seinen Ruf als herausragender Vertreter seiner Komponistengeneration festigte.

Eine Oper für Neapel zu schreiben, war für Verdi doppelt reizvoll. Der Auftrag des Teatro San Carlo, einer der wichtigsten Opernbühnen Italiens, stellte einerseits einen großen Prestigegewinn dar, andererseits gehörte mit Salvadore (Salvatore) Cammarano einer der besten Librettisten der Zeit zum Stammpersonal des Hauses. ${ }^{3}$ Für Gaetano Donizetti hatte Cammarano unter anderem das Textbuch zu Lucia di Lammermoor geschrieben; mehrere Libretti hatte er für Saverio Mercadante verfasst, einen in Neapel besonders beliebten Komponisten, der seit 1840 zudem als Direktor des lokalen Conservatorio San Pietro a Majella wirkte. Für Verdi erwies sich die Begegnung mit Cammarano als folgenreich, denn der Librettist schrieb für ihn nach Alzira die Textbücher zu drei weiteren Opern (La battaglia di Legnano, Luisa Miller und Il trovatore) und skizzierte auf Wunsch des Komponisten eine Oper nach Shakespeares King Lear, ein Projekt, das Verdi viele Jahre lang verfolgte, zu dessen Ausführung es aber nie kam. ${ }^{4}$

Wie im italienischen Opernbetrieb dieser Zeit üblich, stand zu Beginn von Verdis Engagement noch nicht fest, welcher Stoff dem in Auftrag gegebenen Werk zugrunde liegen würde. ${ }^{5}$ Der Vorschlag, Voltaires Tragödie Alzire ou les Américains zur Grundlage zu machen, stammte von Cammarano. Arbeitsökonomische Gründe scheinen dabei eine Rolle gespielt zu haben. Der Librettist hatte sich bereits zuvor mit diesem Drama beschäftigt und dessen Stoff auf seine Operntauglichkeit hin durchdacht. Zwei Jahre vor Verdi schlug er dem renommierten und in Neapel sehr geschätzten Komponisten Giovanni Pacini eine Oper nach Voltaires Drama vor, dieser lehnte aber zugunsten eines anderen Projekts ab. ${ }^{6}$ Cammarano hatte also Ideen für eine Alzira-Oper in der Schublade, als er Verdi, dem jungen Komponisten aus Norditalien, der seinen Platz in der Riege der großen italienischen Opernschöpfer erst noch finden musste, einen Stoff anbieten sollte. Er schickte Verdi ein Szenario (programma), das dieser sofort akzeptierte; mehr noch: Er teilte Cammarano mit, er sei „,contentissimo sotto ogni rapporto“ (,höchst zufrieden in jeder Hinsicht'). ${ }^{7}$ Verdi scheint Voltaires Drama zuvor nicht gekannt zu

3 John N. Black: The Italian Romantic Libretto. A Study of Salvatore Cammarano. Edinburgh 1984.

4 Gary Schmidgall: Verdi's „King Lear“ Project. In: 19 ${ }^{\text {th }}$-Century Music 9: 2, 1985/86, 83-101.

5 Einen knappen Überblick zur „Opernindustrie“ der Verdi-Zeit gibt Anselm Gerhard: Giuseppe Verdi. München 2012, 14-28, vor allem 16-19.

6 Black: Italian Romantic Libretto (Anm. 3), 86-87.

7 Verdi an Cammarano, 23. Februar 1845. In: Carteggio Verdi - Cammarano (1843-1852), hg. von Carlo Matteo Mossa. Parma 2001, 3. 
haben, nahm aber Cammaranos Vorschlag zum Anlass, die Tragödie zu lesen, ${ }^{8}$ und gewann dabei die Überzeugung, dass aus dem Schauspiel „in den Händen Cammaranos eine ausgezeichnete Oper" werden würde. ${ }^{9}$

Ein solcher Gattungstransfer vom gesprochenen zum gesungenen Drama gehörte zu den Selbstverständlichkeiten der italienischen Opernpraxis. Nach Shakespeare war Voltaire derjenige Dramatiker, dessen Werke seit Ende des 18. Jahrhunderts am häufigsten als Stoffquelle für italienische Opern genutzt wurden. ${ }^{10}$ Besonders beliebt waren Voltaires Dramen Zaire, Sémiramis und Tancrède - Gioachino Rossinis Opern nach den beiden letztgenannten Werken (Tancredi und Semiramide) konnten sich beinahe das ganze 19. Jahrhundert hindurch im Repertoire der Opernhäuser halten. ${ }^{11}$ Auch Alzire war bereits vor Verdi und Cammarano mehrfach für die italienische Opernbühne adaptiert worden, insbesondere um die Wende vom 18. zum 19. Jahrhundert, als italienische Komponisten und Librettisten in Abgrenzung von den historischen und mythologischen Geschichten der älteren Opera seria intensiv nach neuen, unverbrauchten Stoffen suchten. Voltaires Dramen waren in Italien schon zu Lebzeiten des Autors bekannt und wurden in unterschiedlichsten Bearbeitungen häufig gespielt, gelesen und diskutiert. ${ }^{12}$ Der Schritt hin zur musiktheatralischen Bearbeitung war um 1800 ein kleiner.

Voltaires 1736 erstmals aufgeführte Tragödie Alzire ou les Américains spielt in Peru um die Mitte des 16. Jahrhunderts. ${ }^{13}$ Zwei Personengruppen stehen einander gegenüber: die spanischen Eroberer, angeführt von dem Gouverneur D. Alvarès, der seine Macht seinem Sohn D. Gusman übergibt, und die „Américains“, das heißt

8 Die erste italienische Übersetzung von Alzire erschien bereits 1737, ein Jahr nach der Pariser Uraufführung. Bis 1797 folgten sieben weitere Übertragungen; vgl. den Abschnitt „Manuscripts and Editions“ in der "Introduction“ zur kritischen Ausgabe des Dramas von T. E. D. Braun in: Les auvres completes de Voltaire - The Complete Works of Voltaire, hg. von Nicholas Cronk [et al.], Bd. 14. Oxford 1989, 1-210, hier 97-98. Welche Ausgabe Verdi benutzte, ist nicht bekannt.

9 „Ho letto la Tragedia di Voltaire che nelle mani di Cammarano diverrà un'eccelente melodramma" (Carteggio Verdi - Cammarano [Anm. 7], 4).

10 Ronald S. Ridgway: Voltairian Bel Canto. Operatic Adaptations of Voltaire's Tragedies. In: Studies on Voltaire and the Eighteenth Century (SVEC) 241, 1986, 125-154. Michele Calella: Die Rezeption der Tragödien Voltaires auf den venezianischen Opernbühnen und das Problem der Tragik in der Oper des späten 18. Jahrhunderts. In: Franz Hauk und Iris Winkler (Hg.): Johann Simon Mayr und Venedig. Beiträge des Internationalen musikwissenschaftlichen Johann Simon Mayr-Symposions in Ingolstadt vom 5. bis 8. November 1998. München und Salzburg 1999, 155-165.

11 Heather Hadlock: Tancredi and Semiramide. In: Emanuele Senici (Hg.): The Cambridge Companion to Rossini. Cambridge [u.a.] 2004, 139-158.

12 Luigi Ferrari: Le traduzioni italiane del teatro tragico francese nei secoli XVII e XVIII . Saggio bibliografico (Revue de littérature comparée. Bibliothèque; 13). Paris 1925. Laurence Macé: La première réception tragique de Voltaire en Italie. In: Euvres \& Critiques 3: 2, 2008, 133-149.

13 Alle Angaben nach der Ausgabe des Dramas von T. E. D. Braun (Anm. 8). In seiner „Introduction“ gibt Braun einen ausführlichen Überblick zur Entstehungsgeschichte des Dramas, die von Voltaire verwendeten historischen und literarischen Quellen sowie zur Aufführungs- und Publikationsgeschichte des Werks. 
die Vertreter der indigenen Bevölkerung Perus, repräsentiert durch den jungen Zamore, „souverain d'une partie du Potoze“, und den älteren Montèze, „souverain d'une autre partie“, sowie dessen Tochter Alzire. Alvarès bemüht sich um eine friedliche Koexistenz mit den Peruanern und gewinnt Montèze für den Plan, Alzire mit Gusman zu vermählen und durch diese Heirat Frieden zwischen den beiden Völkern zu stiften. Im Unterschied zu seinem Vater setzt Gusman aber auf Gewalt und Unterwerfung der Gegner. Trotzdem begehrt er Alzire, die aber liebend mit Zamore verbunden ist. Zamore, der héros des Dramas, kämpft sowohl für die Freiheit seines Volks wie um seine Geliebte, die auf Druck ihres Vaters bereit ist, Gusman zu heiraten. Im Kampf verwundet Zamore seinen Gegner Gusman schwer, im Sterben erweist sich dieser aber doch noch als echter Christ, indem er seinem Feind vergibt.

In Voltaires Tragödie wird der Konflikt zwischen den handelnden Personen und den Welten und Anschauungen, für die sie stehen, in kunstvoll-beredten Alexandrinern ausführlich entfaltet. Wie viele seiner Stücke ist auch dieses ein Ideendrama, in dem vor allem gesprochen, kaum agiert wird. Die Komplexität der Gedankenführung und die Fülle der miteinander kunstvoll verwobenen Handlungsstränge stehen aber im Widerspruch zur Ästhetik der italienischen Oper, die ein „Drama der Affekte" 14 ist und emotionale Momente, die einer Übersetzung in Musik entgegenkommen, in den Mittelpunkt stellt. Bei Voltaire entfaltet sich die Geschichte im narrativen Diskurs, in der Oper hingegen sind die erzählerischen Momente auf wenige Situationen konzentriert, das Hauptgewicht liegt auf der Präsentation weniger affektstarker Szenen. Aufgabe eines Librettisten war es daher, die opernaffinen Elemente der Dramenvorlage zu erkennen und herauszuarbeiten.

Gaetano Rossi, einer der bekanntesten Librettisten des frühen 19. Jahrhunderts, äußerte sich in Briefen an Giacomo Meyerbeer darüber, wie aus einem literarischen Drama ein italienisches Melodramma zu gewinnen ist. Laut Rossi geht es darum, die Vorlage auf ein Handlungsgerüst, eine „ossatura“ (,Skelett'), zu reduzieren und dieses den gattungsspezifischen Gesetzen der Oper anzupassen, das heißt, Szenen auszuwählen, die sich in geschlossene Musiknummern (Arien, Duette, Ensembles et cetera) umformen ließen, oder auch neue Szenen zu erfinden, sollte die Vorlage nicht genügend Anlässe für Musik bieten. ${ }^{15}$ Rossi war der Librettist von Tancredi (1813) und Semiramide (1823), Rossinis Erfolgsopern nach Voltaire; zuvor hatte er bereits dessen Alzire zu einer Oper umgearbeitet, zunächst unter dem Titel Gli americani für Johann Simon Mayr (Venedig, Teatro La Fenice, 1805), dann als Alzira für Nicola Antonio Manfroce (Rom, Teatro Valle, 1810) und schließlich als Il trionfo di Gusmano für Marcos António Portugal (Lissabon,

14 Carl Dahlhaus: Wagners Konzeption des musikalischen Dramas. Kassel [u.a.] 1990, 18.

15 Sabine Henze-Döhring: „Combinammo l'ossatura ...“. Voltaire und die Librettistik des frühen Ottocento. In: Die Musikforschung 36: 3, 1983, 113-127. Zu Rossi vgl. John Black: Rossi, Gaetano. In: Stanley Sadie (Hg.): The New Grove Dictionary of Opera, Bd. 4. London 1992, $52-53$. 
Teatro S Carlos, 1816). ${ }^{16}$ Laut R. S. Ridgway war Rossi auch der Autor der Libretti zu den Alzira-Opern von Giuseppe Nicolini (Genua, Teatro Sant'Agostino, 1796) und von Sebastiano Nasolini (Bologna, Teatro Comunale, 1797; diese Oper stellt eine Bearbeitung von Niccolò Zingarellis Alzira-Oper dar, die 1794 am Teatro della Pergola in Florenz erstmals aufgeführt worden war); ${ }^{17}$ diese Zuweisung ist aber ebenso unsicher wie die Autorschaft Rossis im Fall des Textbuchs zu Francesco Bianchis Alzira (London, King's Theatre in the Haymarket, 1801). ${ }^{18}$

Als Cammarano und Verdi sich mit dem Alzire-Stoff befassten, waren seit der Uraufführung der letzten Alzira-Oper, Portugals Il trionfo di Gusmano, zwar beinahe dreißig Jahre vergangen, der Stoff hatte aber nichts an Reiz verloren, denn Opern, in denen die Eroberung Amerikas durch die Spanier den Handlungsrahmen bilden, waren auf den italienischen Opernbühnen um die Mitte des 19. Jahrhunderts immer noch beliebt. ${ }^{19}$

„Es ist überflüssig, Euch zu sagen, dass Ihr Euch kurz haltet: Ihr kennt das Theater besser als ich" ${ }^{20}$ teilte Verdi dem Textdichter der Alzira mit, und Cammarano folgte dieser Vorgabe. Die meisten Verdi-Opern dauern kaum länger als zwei Stunden, Alzira mit rund anderthalb Stunden Spielzeit, die auf Bitten der neapolitanischen Theaterleitung nachkomponierte Ouvertüre eingeschlossen, galt allerdings schon den Zeitgenossen als „opera, che è brevissima“.21

Cammarano übernimmt das Personal der Voltaire-Tragödie im Wesentlichen und italianisiert die Namen der Protogonisten: Aus Alzire, Zamore, Gusman und Alvarès werden Alzira, Zamoro, Gusmano und Alvaro, nur Alzires Vater Montèze erhält einen neuen, singbareren Namen (Ataliba). Hinzuerfunden sind den Protagonisten zugeordnete Nebenfiguren, die als Stichwortgeber für die Dramaturgie

16 Black: Rossi (Anm. 15), 53.

17 Ridgway: Voltairian Bel Canto (Anm. 10), 153.

18 Sowohl Marita McClymonds (Bianchi, [Giuseppe] Francesco. In: Stanley Sadie [Hg.]: The New Grove Dictionary of Opera, Bd. 1. London 1992, 467) als auch Daniel Brandenburg (Bianchi, Giuseppe Francesco. In: Ludwig Finscher [Hg]: Die Musik in Geschichte und Gegenwart. Allgemeine Enzyklopädie der Musik, Personenteil, Bd. 2. Kassel [u.a.] 1999, Sp. 1564) nennen Rossi als Verfasser des Librettos zu Bianchis Alzira. Im zeitgenössischen Librettodruck des Werks heißt es dagegen: „the fable of this opera has been materially changed from former compositions of this name, and the poetry re-written by S. Buonaiutu“. Angaben nach Francesco Bianchi: Alzira. A Serious Opera in Two Acts. Lambeth 1801, verzeichnet in: Trove. National Library of Australia, http://trove.nla.gov.au/work/152814347 ?q\&versionId=166537980, 29. März 2016. Zu weiteren Alzira-Adaptionen für das italienische Musiktheater vgl. Stefano Castelvecchi: Introduction. In: Le opere di Giuseppe Verdi, Seria 1, Bd. 8: Alzira. Tragedia lirica in 3 acts by Salvadore Cammarano, hg. von Stefano Castelvecchi. Chicago [u.a.] 1994, xi-xxv, hier xiii, Anm. 21.

19 Jürgen Maehder: The Representation of the „Discovery“ on the Opera Stage. In: Carol E. Robertson (Hg.): Musical Repercussions of 1492. Encounters in Text and Performance. Washington und London 1992, 257-287.

20 „È inutile che le dica di tenersi breve: Ella conosce più di me il teatro -“ (Verdi an Cammarano, 23. Februar 1845. In: Carteggio Verdi-Cammarano [Anm. 7], 4).

21 Emanuele Muzio an Antonio Barezzi, 18. September 1845. In: Giuseppe Verdi nelle lettere di Emanuele Muzio ad Antonio Barezzi, hg. von Luigi Agostino Garibaldi. Mailand 1931, 219. 
italienischer Opern typisch sind: Ovando, ein „Duce spagnuolo“, Zulma, „ancella di Alzira“" sowie Otumbo, ein „guerriero americano“.

Cammarano reduzierte Voltaires fünfaktige Tragödie auf eine „ossatura“, aus der er sechs Bilder, das heißt Szenenkomplexe gewann. In den traditionellen Ausgaben der Oper, angefangen mit dem gedruckten Libretto der Uraufführung, verteilen sich diese Bilder auf einen Prolog (ein Bild) und zwei Akte (mit zwei beziehungsweise drei Bildern). In seiner handschriftlichen Partitur bezeichnet Verdi den ersten Teil der Oper dagegen eindeutig als I. Akt, der I. und II. Akt Cammaranos sind bei ihm dementsprechend die Akte II und III. ${ }^{22}$ Cammaranos Bezeichnung der ersten Szene als "prologo" ist allerdings treffender, denn in ihr wird ein Ereignis gezeigt, das dem Hauptgeschehen zeitlich vorgelagert ist und im späteren Handlungsverlauf eine wichtige Rolle spielt. Zu Beginn der Oper wird gezeigt, dass Alvaro in die Hände der Inkas gefallen ist und auf Befehl Otumbos zu Tode gefoltert werden soll. Überraschend tritt der tot geglaubte Zamoro auf und befiehlt, Alvaro freizulassen. Es ist eine Geste an die Adresse der Feinde: Alvaro soll seinen Landsleuten berichten, dass ein Wilder ihm das Leben geschenkt habe. ${ }^{23}$ An diese Gnadengeste erinnert sich Alvaro später, wenn Zamoro Gefangener Gusmanos ist und hingerichtet werden soll. In Voltaires Drama findet sich diese Szene nicht, wohl aber das ihr zugrunde liegende Motiv: In der 2. Szene des II. Akts berichtet Alvarès von einem „héros inconnu“, der ihn vor den rachedurstigen Peruanern gerettet habe. ${ }^{24}$ Im Sinne der auf sinnliche Verdeutlichung zielenden Ästhetik der italienischen Oper macht Cammarano diese Tat sichtbar; aus einer Nebenbemerkung bei Voltaire entwickelt er eine theatralisch wirkungsvolle Szene.

Die weitere Handlung ist in wenigen Sätzen zusammenzufassen: Alvaro übergibt die Statthalterschaft an seinen Sohn Gusmano, dieser fordert die Hand Alziras von Ataliba, der für seine Tochter aber um Bedenkzeit bittet (2. Bild). Alzira träumt von ihrem tot geglaubten Geliebten Zamoro, der plötzlich erscheint, nachdem Ataliba versucht hat, seine Tochter zur politischen Ehe mit Gusmano zu bewegen. Das wiedervereinigte Liebespaar wird von Gusmano überrascht, der Zamoro gefangen nehmen lässt und seine Hinrichtung anordnet. Alvaro erkennt in $\mathrm{Za}$ moro seinen Retter, Gusmano lässt den Inka-Führer auf die Bitte seines Vaters frei, ist aber entschlossen, seinen Rivalen im bevorstehenden Kampf zwischen Spaniern und Peruanern zu besiegen (3. Bild). In der Schlacht wurden die Inkas geschlagen, Zamoro ist wieder Gefangener Gusmanos und soll auf dem Scheiterhaufen sterben. Um ihren Geliebten zu retten, willigt Alzira ein, Gusmano zu heiraten (4. Bild). In einer Höhle im Gebirge beklagt Zamoro den erneuten Verlust Alziras. Von Otumbo erfährt er, welches der Preis für seine Freilassung war. Aufgebracht

22 Die Verdi-Gesamtausgabe folgt dieser Zählung; vgl. Castelvecchi: Introduction (Anm. 18), xxi-xxii.

23 „Fra' tuoi | Ritorna, o vecchio, ed a color, che noi | Chiaman selvaggi, narra | Che ti donò la vita | Un selvaggio." (Tutti i libretti di Verdi, hg. von Luigi Baldacci. Mailand 1975, 122)

24 Voltaire: Alzire ou les Américains (Anm. 8), 146. 
eilt er zurück in die Stadt, um sich an Gusmano zu rächen (5. Bild). Auf dem Hochzeitsfest erscheint Zamoro in spanischer Uniform und verletzt Gusmano tödlich. Dieser verzeiht seinem Gegner im Sterben und gibt Alzira frei (6. Bild).

Dieses aus den Hauptmotiven des Voltaire-Dramas destillierte Handlungsgerüst musste der Librettist so ausfüllen, dass die Protagonisten jenen Raum zur Entfaltung in Arien und Ensembles erhielten, der ihnen der Gattungskonvention gemäß zustand. In den 1830er Jahren etablierte sich in der italienischen Oper ein neuer Ensembletypus mit einer Dreieckskonstellation als Mittelpunkt: ein Liebespaar, bestehend aus Sopran und Tenor, und ein Bariton als Figur, die in Spannung zur Liebesbeziehung der anderen Protagonisten steht, sei es als Nebenbuhler (wie der Conte di Luna in Verdis Il trovatore), sei es als Vater (wie in Rigoletto). ${ }^{25}$ In Alzira bilden die Titelfigur und Zamoro das Liebespaar aus Sopran und Tenor, Gusmano, der Kontrahent, ist der Bariton.

In den Alzira-Opern, die um 1800 entstanden, ist dagegen eine Disposition der Stimmen zu beobachten, die noch der Ästhetik des 18. Jahrhunderts verpflichtet ist. Der auffälligste Unterschied zur späteren Konvention besteht in der Besetzung der Heldenpartie des Zamoro mit einer Sopranstimme. ${ }^{26}$ Vor 1800 wurde die Partie ausnahmslos mit Kastraten besetzt: ${ }^{27}$ Vitale Damiani sang den Zamoro in Niccolò Zingarellis Oper (1794), Girolamo Bravura trat 1797 in den Alzira-Opern von Giuseppe Nicolini und Sebastiano Nasolini in dieser Rolle auf. Nach 1800 setzte sich eine andere Besetzungsstrategie durch. Die Hochzeit der Kastraten war vorbei, nicht zuletzt wohl aufgrund des mehrjährigen Kastratenverbots, das Napoleon über die italienischen Bühnen verhängt hatte. ${ }^{28}$ Die Identifizierung des Helden mit einer hohen Stimme galt aber nach wie vor, doch griff man seit etwa 1810 zunehmend auf Sängerinnen in Männerkostümen zurück, eine Besetzungskonvention, aus der sich ein eigenes Opernrollenfach, das des Contralto musico herausbildete. ${ }^{29}$ Als Nicola Monfroces Fassung von Rossis Alzira-Libretto 1810 ihre erste Aufführung erlebte, sang und spielte Adelaide Malanotte ${ }^{30}$ den Zamoro, auch in allen dokumentierten Folgeaufführungen dieser Oper bis hinein in die

25 Uwe Schweikert: ,Das ewige Dreieck'. Sängerhierarchie, Werkbegriff, Gesangsästhetik, Stimmtypologie, Personenkonstellation und Rollencharaktere. In: Uwe Schweikert und Anselm Gerhard (Hg.): Verdi-Handbuch. Kassel [u.a.] 22013, 140-164.

26 Thomas Seedorf: Heldensoprane. Die Stimmen der eroi in der italienischen Oper von Monteverdi bis Bellini (Figurationen des Heroischen; 1). Göttingen 2015.

27 Angaben nach Roberto Verti (Hg.): Un almanacco drammatico. L'indice de' teatrali spettacoli 1764-1823, 2 Bde. (Saggi e forti / Fondatione Rossini; 2). Pesaro 1996.

28 Seedorf: Heldensoprane (Anm. 26), 62-64.

29 Der Begriff setzt sich zusammen aus der Stimmgattungsbezeichnung contralto und musico, einer seit dem 17. Jahrhundert geläufigen Bezeichnung für Sängerkastraten. Vgl. Heather Hadlock: Women Playing Men in Italian Opera, 1810-1835. In: Jane A. Bernstein (Hg.): Women's Voices across Musical Worlds. Boston 2004, 285-307. Anke Charton: ,prima donna, primo uomo, musico: Körper und Stimme. Geschlechterbilder in der Oper (Leipziger Beiträge zur Theatergeschichtsforschung; 4). Leipzig 2012. Seedorf: Heldensoprane (Anm. 26), 64-66.

30 Für Malanotte komponierte Rossini 1813 die Titelpartie in Tancredi, die zur Paraderolle vieler Vertreterinnen des Contralto musico wurde. 
1820er Jahre wurde die Partie stets mit einer Frau besetzt. ${ }^{31}$ Eine zukunftsweisende Ausnahme bildete die Besetzung dieser Rolle mit einem Tenor - Giuseppe Viganoni - in Francesco Bianchis Alzira, die 1801 für London entstand. ${ }^{32}$

Als Verdi seine ersten Opern komponierte, neigte sich die Tradition des Contralto musico bereits ihrem Ende entgegen. Es gab aber immer noch Sängerinnen, die in Männerrollen auftraten und das Publikum zu begeistern vermochten. $\mathrm{Zu}$ ihnen zählte die Altistin Carolina Vietti, die insbesondere in Venedig eine große Anhängerschaft hatte. Als die Leitung des Teatro La Fenice wegen der Besetzung der geplanten Oper Ernani mit Verdi verhandelte, legte man dem Komponisten nahe, die Titelrolle des Outlaw Ernani als Contralto musico anzulegen, um eine dankbare Partie für die Vietti zu schaffen. Verdi lehnte zunächst ab, lenkte dann ein, um sich schließlich doch vehement gegen die alte Besetzungspraxis zu verwahren und die Rolle des Ernani für einen Tenor zu schreiben, wie es der modernen Praxis entsprach. ${ }^{33}$

Tenor war (und ist) allerdings nicht gleich Tenor. Carlo Guasco, der erste Ernani, wurde, so die zeitgenössischen Kritiker, den dramatischen Zügen seiner Partie nur unzureichend gerecht, vor allem mangelte es ihm für die vielen con forza zu singenden Stellen an klanglicher Durchschlagskraft. Guasco war Vertreter eines älteren Tenortypus, für den sich im Nachhinein der Terminus tenore di grazia oder tenore leggero etablierte. Ihm gegenüber entwickelte sich seit den 1820er Jahren ein anderer Typus, der tenore di forza, der in den 1830er Jahren die Heldenpartien zu erobern begann. ${ }^{34}$

Zum Sängerensemble, für das Verdi Alzira komponierte und dessen stimmlichdarstellerische Potenziale Librettist und Komponist in der Konzeption von Text und Musik berücksichtigten, gehörte der Tenor Gaetano Fraschini. ${ }^{35}$ Geboren und ausgebildet in seiner Heimatstadt Pavia, debütierte Fraschini mit vierundzwanzig Jahren an der Mailänder Scala und sang kurz darauf auch erstmals am Teatro San Carlo in Neapel, das für viele Jahre eine seiner wichtigsten Wirkungsstätten werden sollte. Fraschinis Ruhm gründete auf seiner Darstellung des Sir Edgardo di Ravens-

31 Vgl. die Besetzungsangaben bei Nicola Antonio Manfroce News. Lavori Teatrali, http:// nicolaantoniomanfr.wix.com/manfroce\#!lavori-teatrali-bis/c1kba, 29. März 2016.

$32 \mathrm{Zu}$ Viganonis Auftritt als Zamoro sind keine zeitgenössischen Berichte überliefert. Viganoni war der Lehrer Domenico Donzellis, der in den 1830er Jahren zu den zentralen Protagonisten im Wandel des Tenorgesangs gehörte. Vgl. Giorgio Appolonia: Il dolce suono mi colpi di sua voce. Giuseppe Viganoni da Almenno e i tenori bergamaschi del primo Ottocento. Bergamo 2010. Julian Budden: The Operas of Verdi, Bd. 1: From Oberto to Rigoletto. New York 1973, 138-171.

34 Thomas Seedorf: Heldentenor und Tenore di forza. In: Arnold Jacobshagen (Hg.): Verdi und Wagner. Kulturen der Oper. Köln [u.a.] 2014, 295-305.

35 Giorgio Migliavacca: Gaetano Fraschini: il tenore della transizione da Donizetti a Verdi. In: Moderne Sprachen 44, 2000, 207-232. Dario de Cicco (Hg.): „Amico carissimo ... “. Verdi e Fraschini attraverso alcune corrispondenze. Lucca 2013. Giancarlo Landini: Fraschini, Rossini, Pacini, Petrella e Mercadante. Un problema d'interpretazione ed un tenore lombardo nella fucina del romanticismo napoletano. In: Sergio Martinotti (Hg.): La musica a Milano, in Lombardia e oltre, Bd. 2. Mailand 2000, 251-320. 
wood in Gaetano Donizettis Lucia di Lammermoor. In der zentralen Szene im Finale des II. Akts verflucht Edgardo seine Geliebte Lucia wegen ihrer vermeintlichen Untreue. Fraschini gestaltete diesen Ausbruch („Maledetto sia l'instante | che di te mi rese amante ${ }^{\text {“36) }}$ mit dem immer wieder exponierten und con forza $\mathrm{zu}$ singenden $a^{1}$ stimmlich und darstellerisch so überzeugend, dass er den Beinamen Tenore della maledizione erhielt. Auch in anderen Rollen wie etwa Verdis Ernani konnte Fraschini neue Maßstäbe setzen. Im Gegensatz zu Carlo Guasco verfügte Fraschini über die Klangenergie und Durchschlagskraft, die für eine überzeugende Interpretation dieser Partie notwendig sind. Zeitgenössische Berichte attestieren dem Sänger zudem eine bemerkenswerte stimmliche Ausdauer, die es ihm ermöglichte, auch in Ensemblesätzen stets mit voller Stimme zu singen. Pendant zur dramatischen Eruptivität war Fraschinis Vermögen, intime Momente mit größter Intensität und feinsten Klangschattierungen zu gestalten. Vor allem Kraft und Ausdauer Fraschinis und das Timbre seiner Stimme wurden in der zeitgenössischen Presse als dezidiert männlich wahrgenommen und beschrieben. Dieser „new sound of masculinity“37 entsprach dem intensiv geführten Diskurs über ein neues Verständnis von Männlichkeit und Weiblichkeit in den vorrevolutionären 1840er Jahren, der in der Konzeption vieler Verdi-Opern dieser Zeit Spuren hinterlassen hat.

Mit Fraschini hatte Verdi die Möglichkeit, Zamoro auch auf stimmlicher Ebene als eroe im Sinne seiner Zeit zu inszenieren, als einen Helden, der ein Kollektiv sowohl anführt wie aus ihm herausragt. Verdi nutzte diese Möglichkeit auch an einer Stelle, an der die Herausstellung des Helden im Libretto ursprünglich gar nicht vorgesehen war. Cammarano dichtete für den Schluss des Prologs einen Kriegsgesang der Peruaner, der allein dem Chor vorbehalten ist. Er fungiert als Pendant zum erregten Rachechor der Introduzione („Muoia, muoia coverto d'insulti“), sodass Zamoros große Szene mit der Arie „Un Inca... eccesso orribile!“ im Zentrum des Prologs (I. Akt in Verdis Zählung) von zwei Chören formal gerahmt wird. Diese Fassung findet sich noch im gedruckten Libretto zur Uraufführung. ${ }^{38}$

Verdi verwendet diesen Text - zwei Strophen zu je vier Versen im Versmaß eines Decasillabo - als Material für ein Finale, in dem Zamoro sich teils mit dem Chor vereinigt, teils aus dem Kollektiv heraushebt. ${ }^{39}$ Nachdem er Alvaro das Leben geschenkt und damit demonstriert hat, dass auch ein "selvaggio", 40 ein vermeintlich ,Wilder', barmherzig sein kann, zeigt Zamoro sich jetzt als zum Kampf bereiter Krieger. Verdi inszeniert in diesem Gesang auch seinen Zamoro-Sänger

36 Tutti i libretti di Donizetti, hg. von Egidio Saracino. Mailand 1993, 826.

37 Susan Rutherford: „Il grido dell'anima“, or „Un modo di sentire“. Verdi, Masculinity and the Risorgimento. In: Studi verdiani 19, 2005, 107-121, hier 109.

38 Pierluigi Petrobelli: Thoughts for Alzira. In: Pierluigi Petrobelli: Music in the Theater. Essays on Verdi and Other Composers. Princeton, NJ 1994, 75-99.

39 Ebd., 87, Abb. 4.1.

40 Vgl. Anm. 23. 

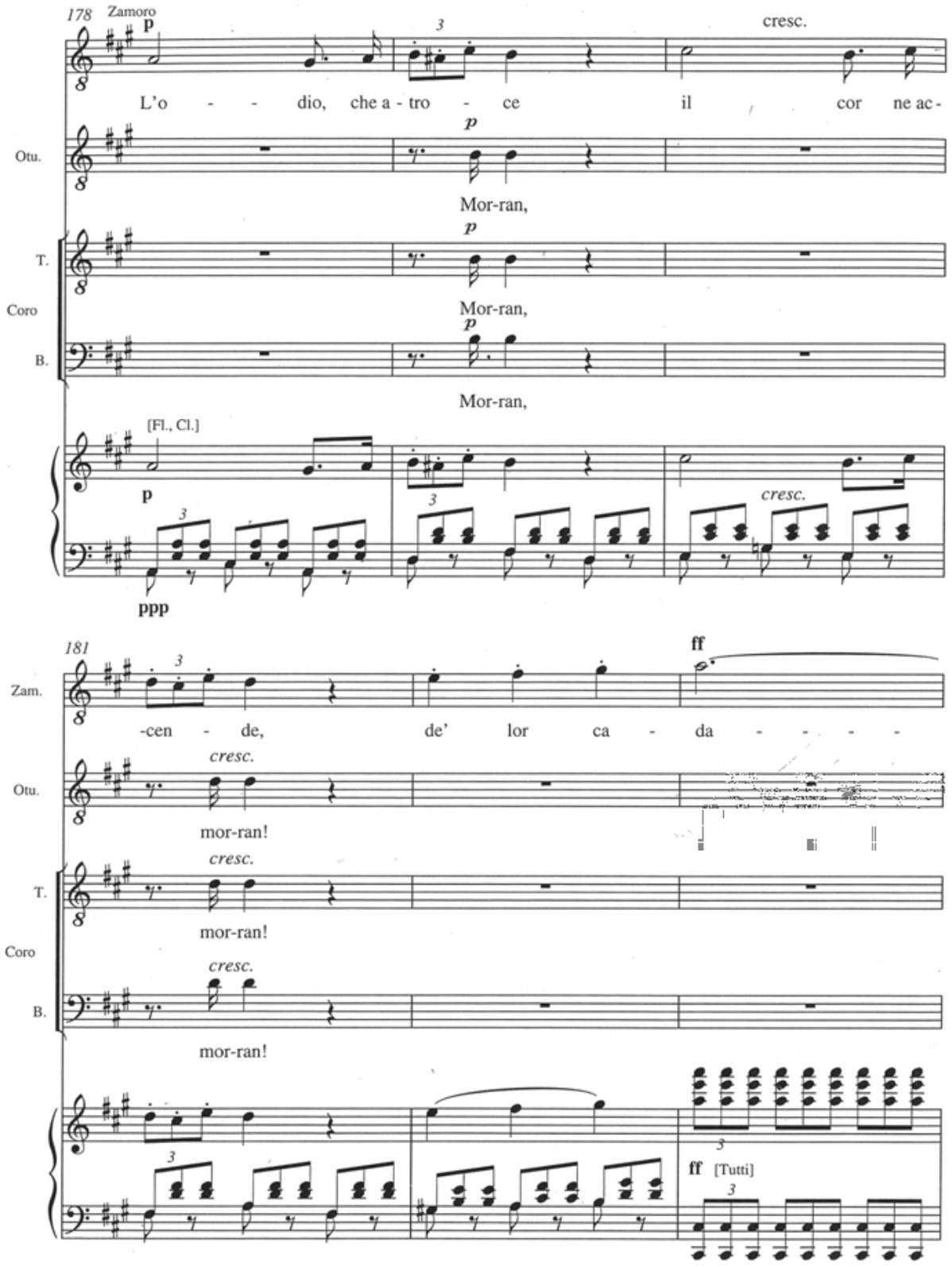

Notenbeispiel 1: Giuseppe Verdi: Alzira, Atto primo, N. 2, Scena Zamoro. 

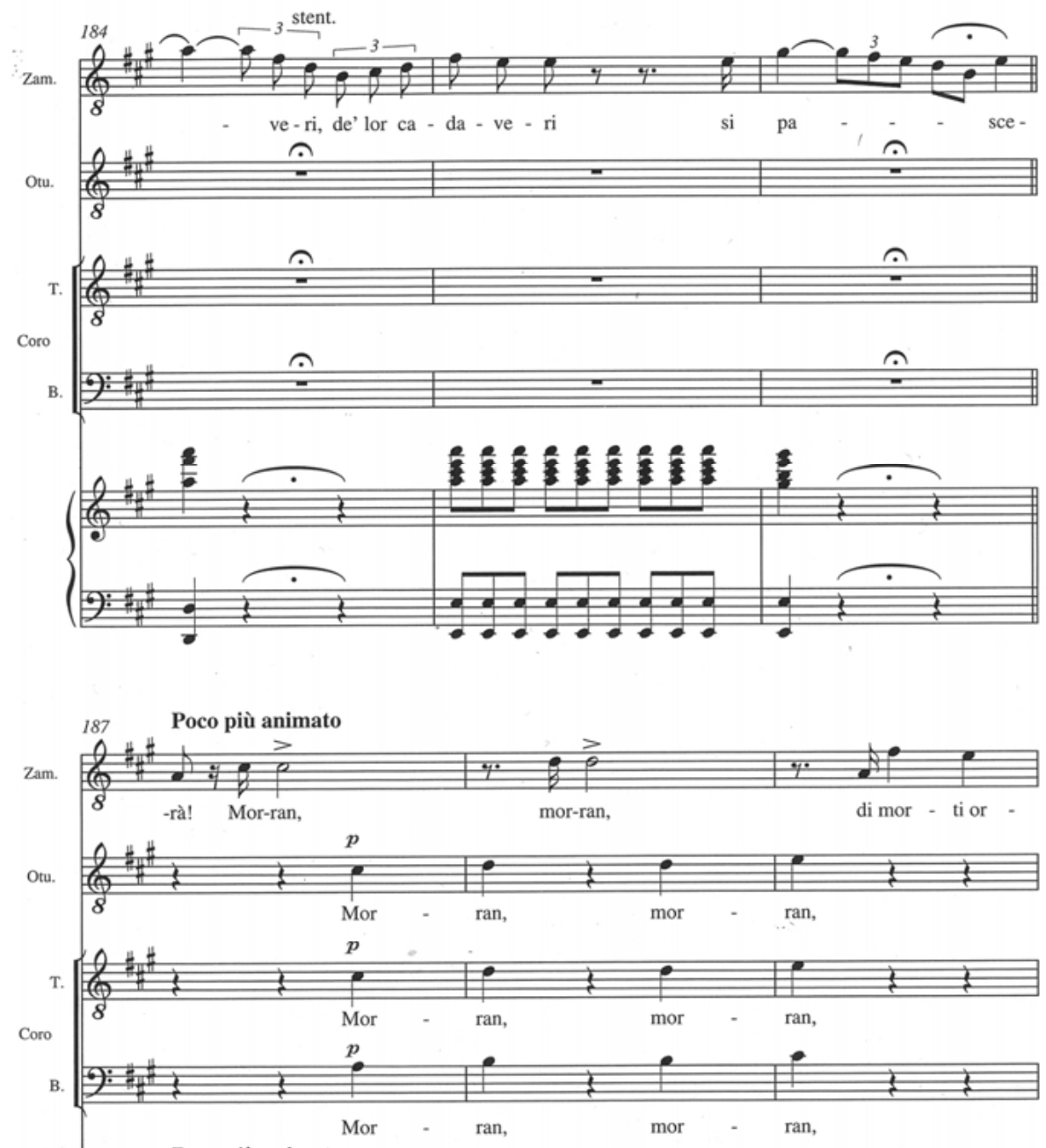

Poco più animato

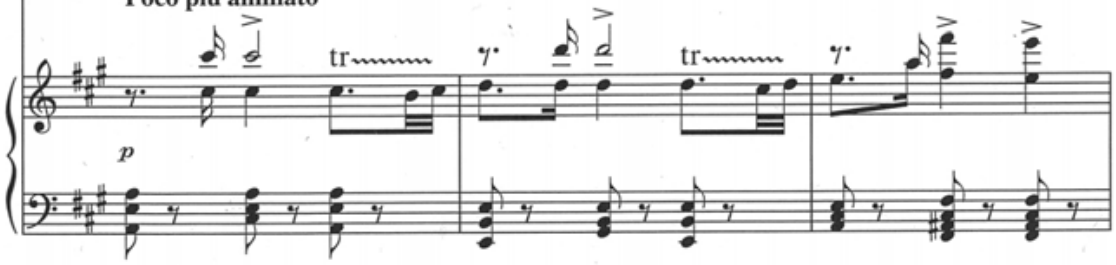

(Notenbeispiel 1: Fortsetzung) 


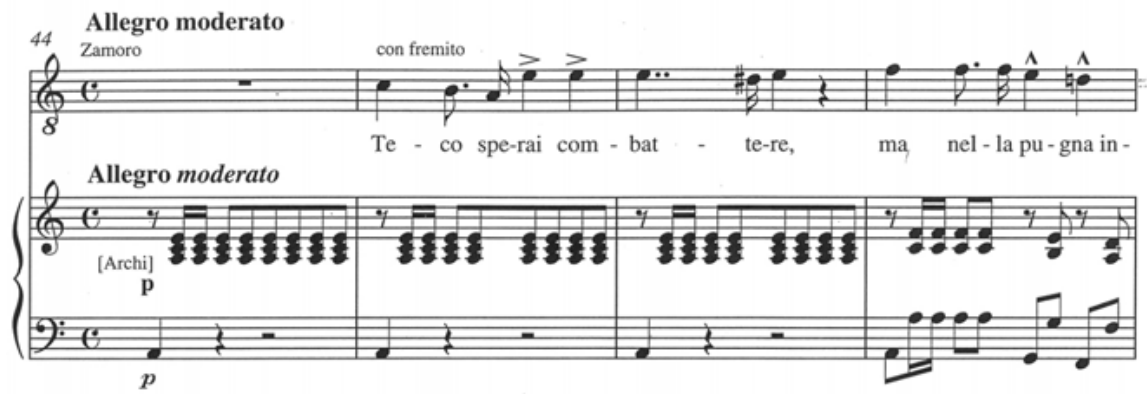

Zam.
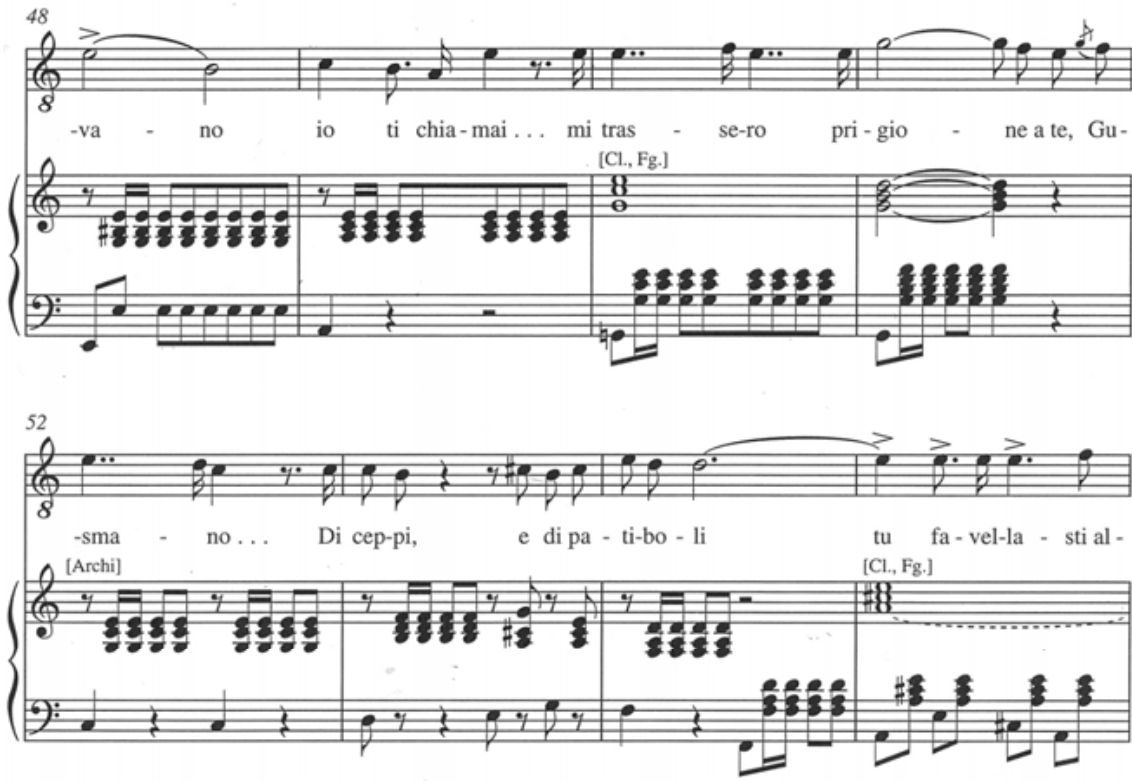

Zam.

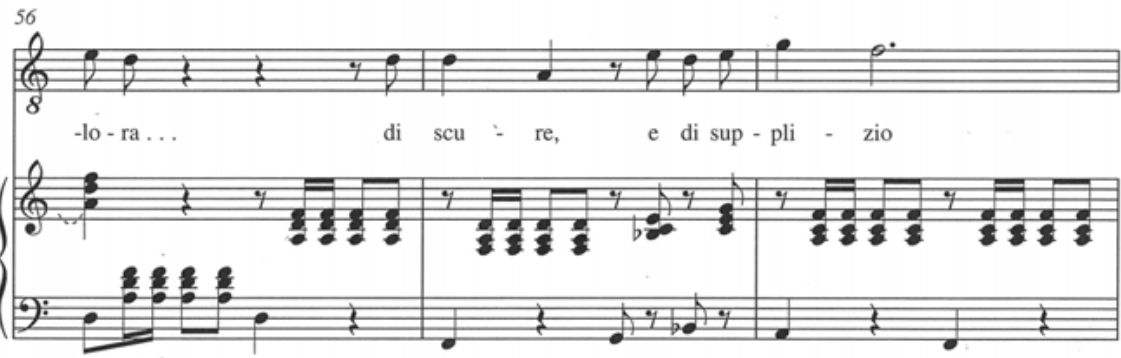

Notenbeispiel 2: Giuseppe Verdi: Alzira, Atto secondo, N. 7, Finale [Secondo]. 

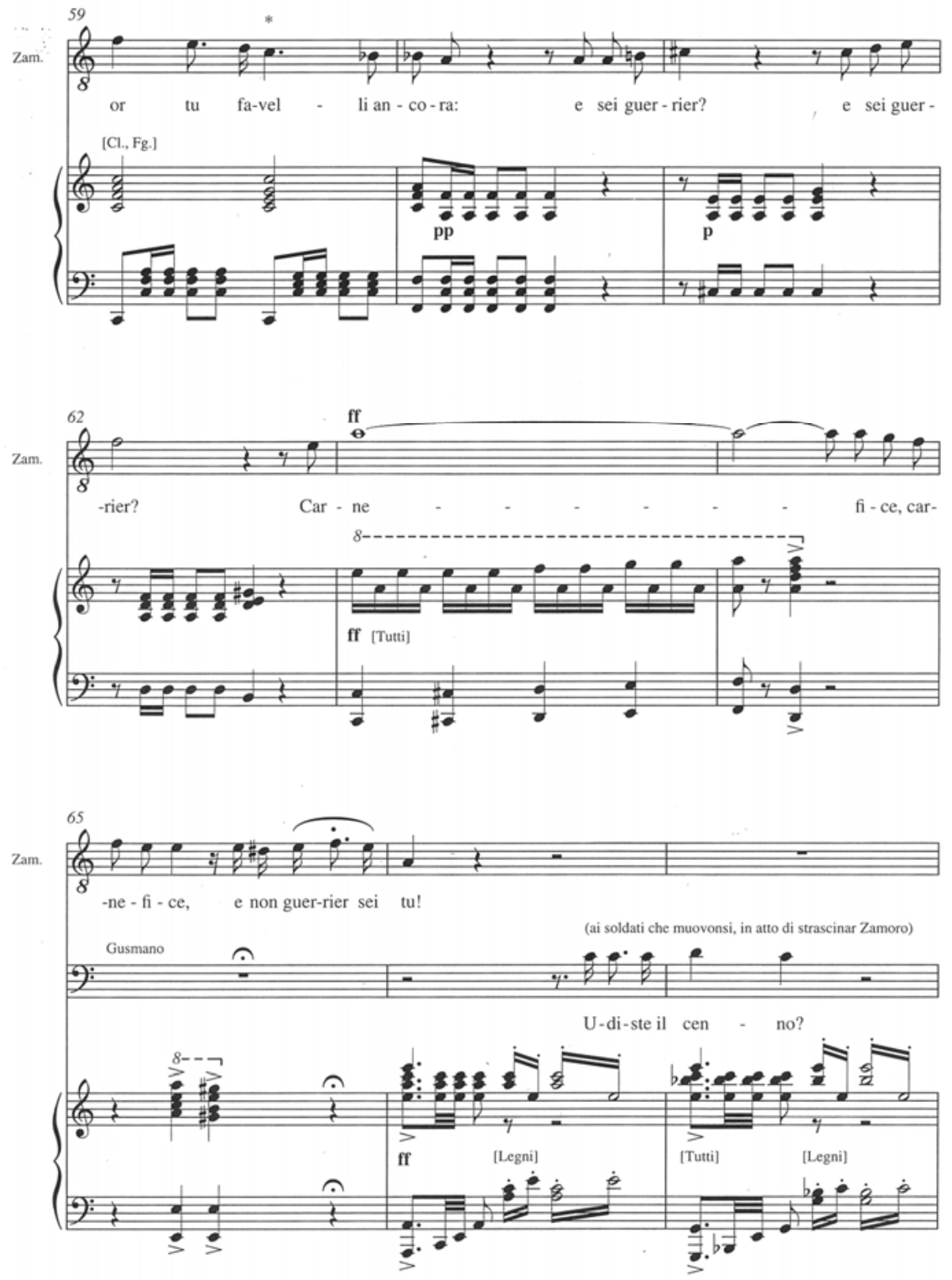

(Notenbeispiel 2: Fortsetzung) 
Fraschini, dessen Fähigkeit, hohe Töne mit hoher Energie zu singen, er hier eindrucksvoll zur Geltung kommen lässt (Notenbeispiel 1). In Takt 178 beginnt mit „L'odio, che atroce“ ein vokaler Aufschwung, der auf einem im Fortissimo lang auszuhaltenden hohen $a$ in Takt 183/184 kulminiert, das heißt, einen Ton exponiert, den Fraschini besonders eruptiv zu gestalten vermochte.

Vergleichbare vokale Inszenierungen der heroischen Haltung Zamoros, zu denen Verdi durch das sängerische Potenzial Fraschinis angeregt wurde, finden sich an zwei weiteren Stellen der Oper. Im Finale des II. Akts (in Verdis Zählung) steht Zamoro seinem Gegner Gusmano gegenüber, der seinen Feind und Rivalen hinrichten lassen will. Zamoro sieht darin ein unwürdiges Verhalten: „Henker bist du, kein Krieger!“, wirft er Gusmano entgegen (Notenbeispiel 2). ${ }^{41}$ Wieder ist es ein mit hoher Energie zu singendes $a^{1}$, das den Höhepunkt markiert. Ein letztes Mal begegnet das herausgehobene hohe $a$ im Allegro-Teil von Zamoros Arie im III. Akt (wiederum nach Verdis Zählung), wo Verdi es mit besonderem Nachdruck auf den Wörtern sangue und morte einsetzt - ein kraftvoller Ton als vokale Klangchiffre des Heroischen.

Indem er die Partie des Zamoro für einen Tenor neuer Prägung, einen tenore di forza schrieb, setzte sich Verdi deutlich von jenen Komponisten ab, die vor ihm Voltaires Alzire zur Grundlage einer Oper gemacht hatten und für die Stimme des Helden einen ,Heldensopran ${ }^{\triangleleft 2}$ wählten. Sie folgten darin einer beinahe zwei Jahrhunderte alten Tradition, deren Endpunkt ein Werk wie Verdis Alzira markiert.

Was Verdi aber mit seinen Vorgängern verbindet, ist die weitgehende Nivellierung der exotischen Elemente, die ein Stoff, der in Südamerika spielt, ja eigentlich bietet. Schon bei Voltaire spielt der Schauplatz nur eine nachgeordnete Rolle: „Alzire does not abound in local colour". ${ }^{43}$ Ein Kritiker der Uraufführung von Verdis Alzira hob zwar den „bel contrasto dei costumi americani ed europei“44 hervor, doch im Gegensatz zu den Kostümen findet sich in der Musik keine Unterscheidung zwischen den beiden Personengruppen. Die kompositorischen Mittel, die Verdi einsetzt, um den Inka Zamoro zu charakterisieren, sind keine anderen als jene, mit denen er dessen Gegenspieler Gusmano ausstattet. ${ }^{45}$

Für Ästhetik und Dramaturgie der italienischen Oper des Primo Ottocento konnten exotistische Elemente allenfalls eine periphere Bedeutung haben, denn im Mittelpunkt standen die emotionalen Konflikte der Protagonisten. Zamoro in Verdis Alzira ist, zugespitzt, vor allem der Tenor, dem in der Dreiecksbeziehung mit Sopran (Alzira) und Bariton (Gusmano) die Rolle des um die Geliebte Kämp-

41 „Carnefice, | E non guerrier sei tu!“

42 Zu diesem Begriff vgl. Seedorf: Heldensoprane (Anm. 26), 7-11.

43 Braun: Introduction (Anm. 8), 9.

44 Omnibus: NAPOLI. - Teatro San Carlo. - Alzira. In: Teatri Arti e Letterature 23, Bd. 43, Nr. 1125, 28. August 1845, 217.

45 Erst in Aida (1871) integriert Verdi exotistische Elemente als Grundfarbe in die Partitur eines Werks. Fabrizio Della Seta: ,O cieli azzurri': Exoticism and Dramatic Discourse in Aida. In: Cambridge Opera Journal 3, 1991, 49-62. 
fenden zufällt. Dieses Muster liegt den meisten italienischen Opern dieser Zeit zugrunde, es wird in jedem Werk in immer neuen Varianten, zu denen auch exotische Schauplätze zählen können, durchgespielt.

Nachreis der Notenbeispiele

Notenbeispiel 1: Giuseppe Verdi. Alzira. Tragedia lirica in tre atti. Riduzione per canto e pianoforte condotta sull'edizione critica della partitura a cura di Stefano Castelvecchi. Chicago/Mailand 2002, 44-45.

Notenbeispiel 2: Giuseppe Verdi. Alzira. Tragedia lirica in tre atti. Riduzione per canto e pianoforte condotta sull'edizione critica della partitura a cura di Stefano Castelvecchi. Chicago/Mailand 2002, 114-115. 
